\title{
MODIFYING AND EXPANDING THE SIMULATION OF WEAR IN THE SPHERICAL JOINT WITH A POLYMERIC COMPONENT OF THE TOTAL HIP PROSTHESIS
}

\author{
UDC 617.582
}

\section{Vladimir Pakhaliuk, Aleksandr Poliakov, Mikhail Kalinin, Yevgenii Pashkov, Pavel Gadkov}

Sevastopol State University, Laboratory of Biomechanics, Sevastopol, Russian Federation

\begin{abstract}
The existing model of wear, based on the classical Archard equation, in the spherical joint of a total hip prosthesis comprising an acetabular cup of ultra-high molecular weight polyethylene (UHMWPE) in combination with a metal or ceramic femoral head is modified and expanded. With this model, studies are conducted using the finite element analysis in terms of cumulative linear and volumetric wear for the ISO 14242-1 demands and additionally for the conditions during walking gait. Also they are carried out for the head diameter of $28 \mathrm{~mm}$ at the constant and the variable wear factor, where the variable wear factor is adopted from the modified formula for the dependence on the contact pressure.
\end{abstract}

Key Words: Total Hip Prosthesis, Wear, Finite Element Simulation, Spherical Joint

\section{INTRODUCTION}

It is widely recognized in medical practice that the minimizing of wear and wear debris is crucial in extending the lifespan of artificial joints [1], where the main factor causing failure of the implant is the loosening of its components due to the impact of wear products and reduced thereby the locking of them in the bone tissues. Considering that currently there is a serious trend towards younger patients with indications to be in need of a hip replacement, it is obvious that a lifespan of such prosthesis must be extended up to a maximum of 20 years or more. In this regard, various attempts allowing enhancing

Received October 03, 2016 / Accepted November 16, 2016

Corresponding author: Vladimir Pakhaliuk

Sevastopol State University, Laboratory of Biomechanics, Universitetskaya 33, 299053 Sevastopol, Russian Federation

E-mail: vpakhaliuk@gmail.com 
the durability of total hip replacements (THR) are undertaken. For example, new designs are created on the basis of a systematic approach, taking into account the mutual impact of many factors, both structural and operational [2]. To test the engineering solutions and evaluate durability of the existing and newly created THR, the specialized devices to perform a wide range of tests for wear are used, both for the tests regulated by standards and considering the nature of the non-standard behavior of the patient [3]. Nevertheless, the problem of accurate assessment of the wear parameters of the implant friction surfaces at the design stage is of current interest. This is especially important for modern total hip replacements, which include the acetabular cup of ultra-high molecular weight polyethylene (UHMWPE) in combination with a metal or ceramic femoral ball head.

For preliminary wear evaluation of the sliding surfaces, including modified, the finite element analysis and various wear models (see, e.g., [4, 5] and other sources) are now widely used. In particular, for the THR with a UHMWPE polymeric component the wear simulation is based on the classical Archard equation together with all other studies reported in literature referred to, for example, in $[6,7,8]$, where the wear factor is taken as a constant value. In [5], the polymer wear in terms of cumulative linear and volume wear when the wear factor is chosen to be a function of contact pressure was first evaluated. Herewith, various known wear factor dependencies on the contact pressure were investigated as contained in $[9,10,11]$. Since the contact pressure in the above formulae is a power function with a negative degree, these relations give a decrease in wear factor depending on the increase of a contact pressure, which means a reduction of wear values that are not uniquely consistent with the physics of the process. Conversely, the zero value of contact pressure can lead to uncertainty in the calculations (division by zero), so this case requires limiting it by some small magnitudes, which can lead to a certain error in the calculations. Moreover, study [5] is based only on the demands of ISO 14242-1 standard on the loading and angular femoral movement profiles in the joint, as well as a standard design of the implant head with diameter of $32 \mathrm{~mm}$. Loading and displacement parameters for wear-testing machines and corresponding environmental conditions for test are regulated in the given standard. At the same time, in [7], at a constant wear factor, there was performed the research for the head of $32 \mathrm{~mm}$ and also for standard head diameter of $28 \mathrm{~mm}$. Also, it was conducted under conditions during walking gait when the hip implant experiences three-dimensional load and motion patterns at a gait cycle according to the angular positions measured by Jonhston and Smidt, and load measured by Paul [7]. Herewith, these patterns differ from those of the ISO 14242-1 standard.

The objective of this work is to modify the existing wear model and expand the results obtained in [5] by performing simulations using the finite element analysis for the ISO 14242-1 demands and additionally for the conditions during walking gait [7]. Likewise, it is to be carried out for a head diameter of $28 \mathrm{~mm}$ in terms of cumulative linear and volumetric wear at a constant and variable wear factor, where the variable wear factor is adopted from the modified formula for the dependence on the contact pressure. 


\section{MATERIALS AND METHODS}

The model of the hip joint prosthesis sliding couple contains a solid femoral ball head of cobalt-chromium alloy or ceramics (alumina or zirconia) with widely used standard diameters of $32 \mathrm{~mm}$ and $28 \mathrm{~mm}$ employed against a soft (UHMWPE) acetabular cup [5]. The radial clearance between them makes up 0.15 $\mathrm{mm}$. The elasticity modulus and Poisson's ratio were chosen as $1.4 \mathrm{GPa}$ and 0.46 , respectively, for the cup and $210 \mathrm{GPa}$ and 0.3 , respectively, for the head [7]. The right hip joint, that corresponds to ISO 14242-1 test standard, is defined in anatomically fixed coordinates $x^{\prime} y^{\prime} z^{\prime}$ and shown in Fig. 1. In the simulation of wear, it is proposed to use a simplified coordinate system $X Y Z$ fixed to the cup and placed in its center, see Fig. 2. The movable coordinate system used for the Euler angles coincides with the center of the cup; it is placed in the center of the head and fixed to the head. The head has three rotational degrees of freedom, known as FE (flexion-extension), AA (abductionadduction), and IOR (inward-outward rotation) which correspond to the angular movements profiles in Fig. 3. Graphs of resultant load vector

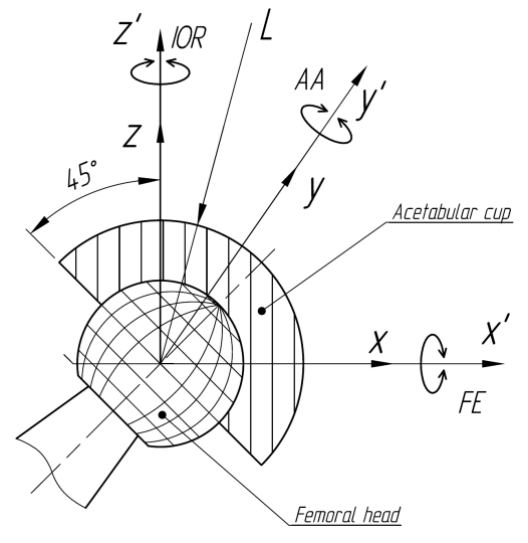

Fig. 1 Front view of the right hip joint with the specified directions of rotation ( $L$ is a resultant load vector) $F_{\text {res }}$ and of its components $F_{X}, F_{Z}$ as projections of two coordinate axes $X$ and $Z$ are shown in Fig. 4. These profiles are related to ISO 14242-1 standard. The profile of the resultant load vector is in the anatomical coordinate system within one gait cycle, which corresponds to the period of time of 1s. The profiles during walking gait of angular femoral positions measured by Jonhston and Smidt are depicted in Fig. 5, and patterns of load, measured by Paul, in Fig. 6 [7].

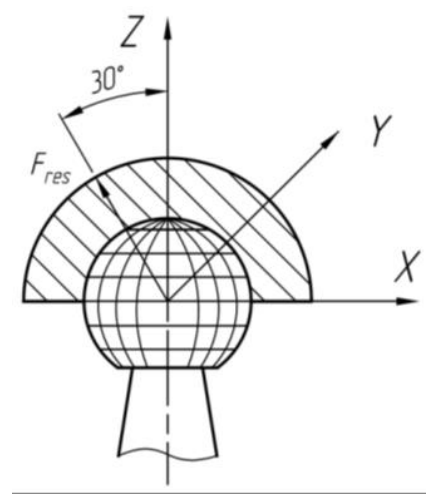

Fig. 2 A simplified coordinate system $X Y Z$ 


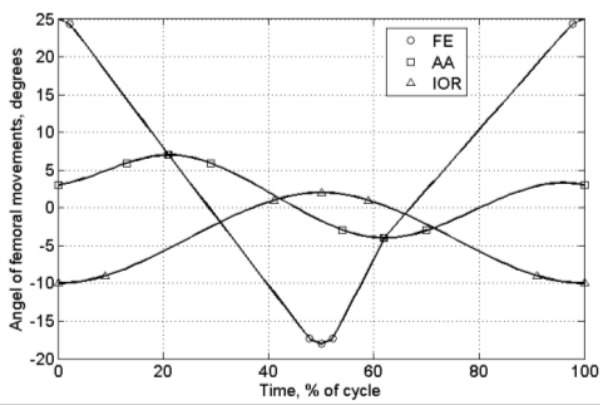

Fig. 3 Graphs of angular movements of the femoral head according to ISO 14242-1

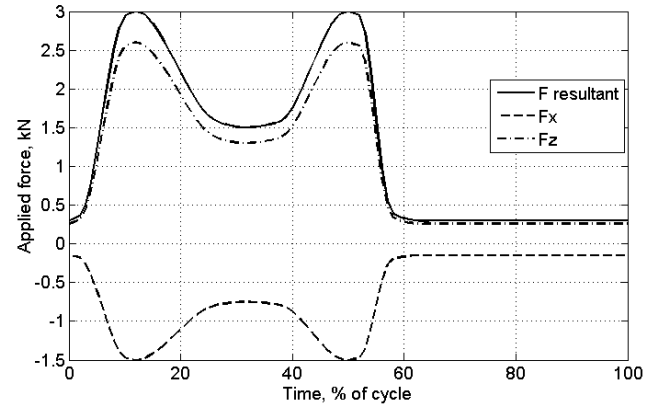

Fig. 4 Graphs of the resultant load vector and its two-dimensional components according to ISO 14242-1

The wear simulation was based on the classical Archard equation [6, 7]. Until now, the total interaction for ideal uniformly loaded isotropic surfaces with a nominal contact pressure in the linear elastic condition is usually adopted for description by the relation:

$$
H=k_{w} \sigma S,
$$

where $H$ is wear depth, $k_{w}$ is a constant empirical wear factor, $\sigma$ is a contact pressure, $S$ is a sliding distance.

In paper [6], relation (1) is proposed in a discrete kind in the parametric form for the evaluation of variables of THR mechanical design and described as following:

$$
\Delta H(\theta, \varphi)=\sum_{i=1}^{n} k_{w} \sigma\left(\theta, \varphi, t_{i}\right) \Delta S\left(\theta, \varphi, t_{i}\right)
$$

where $\Delta H(\theta, \varphi)$ is an accumulative local linear wear depth at the contact surface in a spherical coordinate system, $\sigma\left(\theta, \varphi, t_{i}\right)$ is a normal contact pressure between the counterface surfaces at the same point of time instant $t_{i}$ of the gait cycle, $\Delta S\left(\theta, \varphi, t_{i}\right)$ is an increment of the arc sliding distance between the adjacent measuring points under the same conditions. Wear factor $k_{w}$ depends on the material, nature of the surface and, as was found, the nominal contact pressure.

For a given material combination, the value of constant wear factor in [6, 7] was adopted as $1.066 \cdot 10^{-6} \mathrm{~mm}^{3} /(\mathrm{Nm})$. But, for example, in [10], the wear factor was proposed in a variable form as an empirical relationship with normal contact pressure:

$$
k_{w}=2 \cdot 10^{-6} \sigma^{-0,84},
$$

and in [11] as the next relationships:

$$
\begin{aligned}
& k_{w}=2.7 \cdot 10^{-6}\left(p / p_{\text {ref }}\right)^{-0.57}, \text { when } p / p_{\text {ref }} \leq 2.53 \\
& k_{w}=6.0 \cdot 10^{-6}\left(p / p_{\text {ref }}\right)^{-1.44}, \text { when } p / p_{\text {ref }}>2.53
\end{aligned},
$$

where $p$ and $p_{\text {ref }}=1.1 \mathrm{MPa}$ are the actual nominal contact pressure and the same for the running-in condition, respectively. 
To avoid a power function with a negative degree at using formula (3), Eq. (1) can be modified as following:

$$
H=k_{w} \sigma S=\frac{2 \cdot 10^{-6}}{\sigma^{0.84}} \sigma S=2 \cdot 10^{-6} \sigma^{0.16} S,
$$

where the value of $2 \cdot 10^{-6}$ is a proportionality factor in this case.

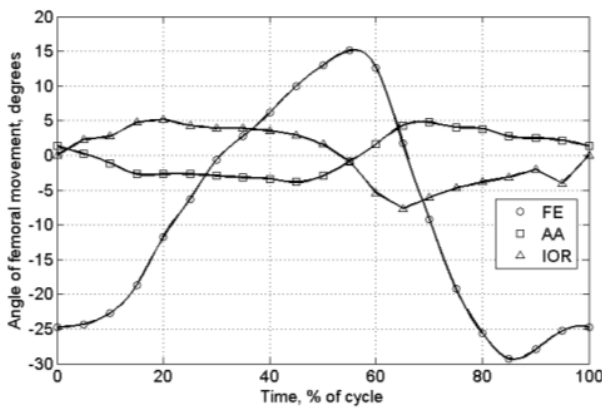

Fig. 5 Graphs of angular femoral positions measured by Jonhston and Smidt during walking [7]

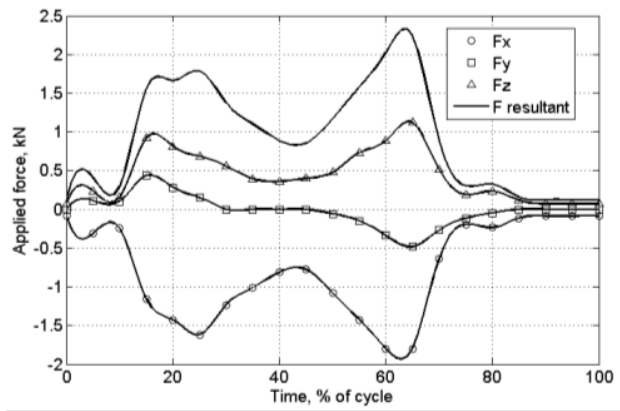

Fig. 6 Graphs of three-dimensional patterns of load measured by Paul during walking [7]

Also, Eq. (1) can be modified considering Eq. (4). Resultant formula (5) is already a power function with a positive degree, and in this case, there is an opportunity to avoid the division by zero at calculations by Eq. (2).

Determining sliding track length $\Delta S\left(\theta, \varphi, t_{i}\right)$ on the surface of the cup is carried out in [5]. There, marker point $K_{0}$ is fixed to the head; one set of rotation angles $\left(\alpha_{\mathrm{i}}, \beta_{\mathrm{i}}, \gamma_{\mathrm{i}}\right)$, corresponding to an anatomical sequence of rotations $\mathrm{FE} \rightarrow \mathrm{AA} \rightarrow \mathrm{IOR}$, is sequentially rotated with Euler rotation angels from the initial position described by vector $\mathbf{r}_{0}$ to a new position $K_{i}$ described by vector $\mathbf{r}_{\mathrm{i}}$ along the sliding track on the cup surface according to the relationship with rotation matrix $\mathbf{R}_{x y z}\left(\alpha_{i}, \beta_{i}, \gamma_{i}\right)$ :

$$
\mathbf{r}_{\mathbf{i}}=\mathbf{R}_{x y z}\left(\alpha_{i}, \beta_{i}, \gamma_{i}\right) \mathbf{r}_{\mathbf{0}}
$$

Then the increment of a sliding track length can be defined with sphere radius $R$ as:

$$
\Delta S\left(\theta, \varphi, t_{i}\right)=R \cdot \arccos \left(\frac{\mathbf{r}_{\mathbf{i}+\mathbf{1}} \mathbf{r}_{\mathbf{i}}}{\left|\mathbf{r}_{\mathbf{i}+\mathbf{1}}\right| \cdot\left|\mathbf{r}_{\mathbf{i}}\right|}\right)
$$

Numerical simulation of wear is carried out using the finite element analysis with ANSYS and MATLAB software and presented in details in [5]. This process can be described in short as follows.

The model of implant couple is created in ANSYS software and meshed with hexagonal finite elements (bricks and wedges) that will improve the accuracy of the calculation (Fig. 7). Then, the contact surface is created, and key points, surfaces and volumes are deleted from the model. It then allows moving the contact surface nodes at the wear calculation. As to the boundary conditions, the outer surface of cup is fully 
constrained through its corresponding nodes. During the first simulation, the coordinates of the contact surface nodes are determined in ANSYS and stored in a text file. The gait cycle is divided into 25 equal time intervals. And in MATLAB software using the indicated text file, the length of tracks in these areas with angular movements according to Fig. 3 or Fig. 5 is determined and also stored in a text file.

Further computational operations are conducted only in the ANSYS software by custom code for all of 25 time intervals in a loop mode than the computational complexity is reduced in the simulation of wear over a long period of time. At the beginning of each cycle, by solving the contact problem of 3-D surface-to-surface, the values of normal contact pressure $\sigma\left(\theta, \varphi, t_{i}\right)$ at the nodes on the contact surface are defined by the corresponding values of load patterns for this interval according to Fig. 4 or Fig. 6. Fig. 8 illustrates the distribution of contact pressure for the first cycle of third interval of gait corresponding to a maximum resultant load of $3 \mathrm{kN}$ according to its pattern in Fig. 4.

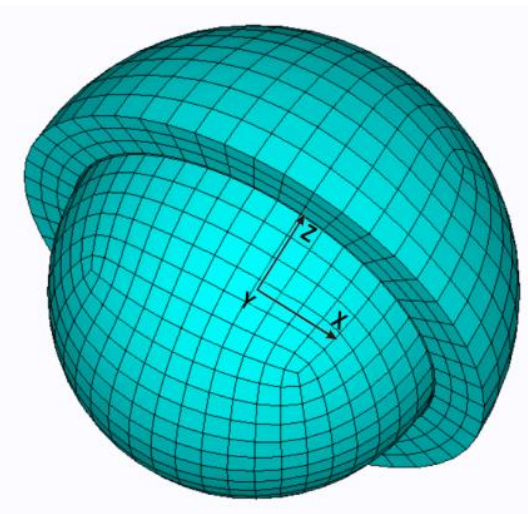

Fig. 7 The model of implant couple with hexagonal finite elements

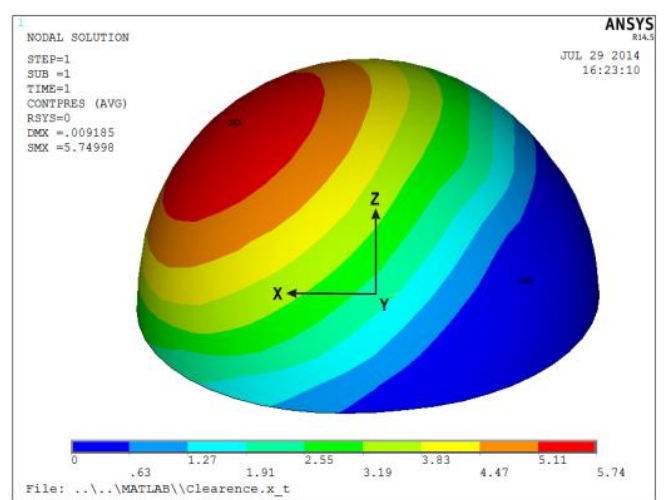

Fig. 8 The distribution of contact pressure for the first cycle of third interval of gait corresponding to a maximum resultant load of $3 \mathrm{kN}$ according to ISO 14242-1

Thereafter, by Eq. (2) considering Eq. (5), local wear depth increment $\Delta H(\theta, \varphi)$ is determined in each of the nodes found at the contact surface. By summing the local depth increments of wear, determined at each interval, we can obtain a distribution of the cumulative linear wear of the cup surface at one gait cycle and the volumetric wear, as the volume of part of the material is removed out of the cup. The results obtained are placed in a text file. Then the actual geometry of the bearing surface of the cup is adjusted by moving the nodes by the amount of linear wear derived from the specified text file and a new contact surface is generated. After that, the specified computing sequence is repeated in a loop mode to perform the required number of cycles corresponding to a predetermined number of gait cycles. Each of the simulations is carried out up to 20 million gait cycles [7], that corresponds to about 20 years of the implant lifespan, with increment of $N_{0}$ cycles. To increase the accuracy of calculations and reduce the PC operating time, before the moving of contact surface nodes, the linear and volumetric wear, obtained at the end of a gait cycle, are here determined by simply multiplying the wear during one cycle by $N_{0}$ cycles. 


\section{RESULTS}

The studies to determine the impact of magnitude of increment $N_{0}$ on the precision of calculations with a head diameter of $32 \mathrm{~mm}$ and according to ISO 14242-1 demands were performed. The investigated increments were of $0.2,0.4$ and 1.0 million cycles. It turned out that at a constant wear factor, the linear wear in the case of these increments is almost the same and the volumetric wear differs only at increments of 1.0 million cycles down by about $6 \%$ (Fig. 9). At a variable wear factor, the linear and volumetric wear differ only at using the increments of 1.0 million cycles down by about $14 \%$ and $10 \%$, respectively (Fig. 10). This is likely derived from the fact that at the smaller increment value a higher magnitude of error is accumulated at each movement of the contact surface. Obviously, this error can be decreased with increasing the mesh grids, but this greatly increases the PC operating time. Therefore, knowledge of the magnitude of this error allows accounting it in the evaluation of the simulation results. In order to reflect more accurately the nature of change of the wear parameters obtained as a result of simulation, the present study was carried out in increments of $N_{0}=0.4$ million cycles.
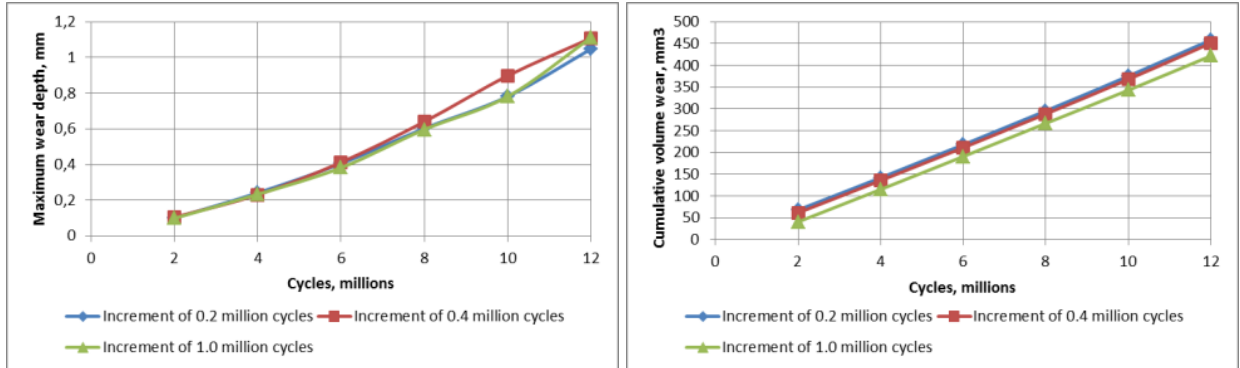

Fig. 9 Graphs on the impact of magnitude of increments of 0.2, 0.4 and 1.0 million cycles on precision of calculations with a head diameter of $32 \mathrm{~mm}$ and according to ISO 14242-1 demands at a constant wear factor $1.066 \cdot 10^{-6} \mathrm{~mm}^{3} /(\mathrm{Nm})$ in terms of the cumulative linear and volumetric wear
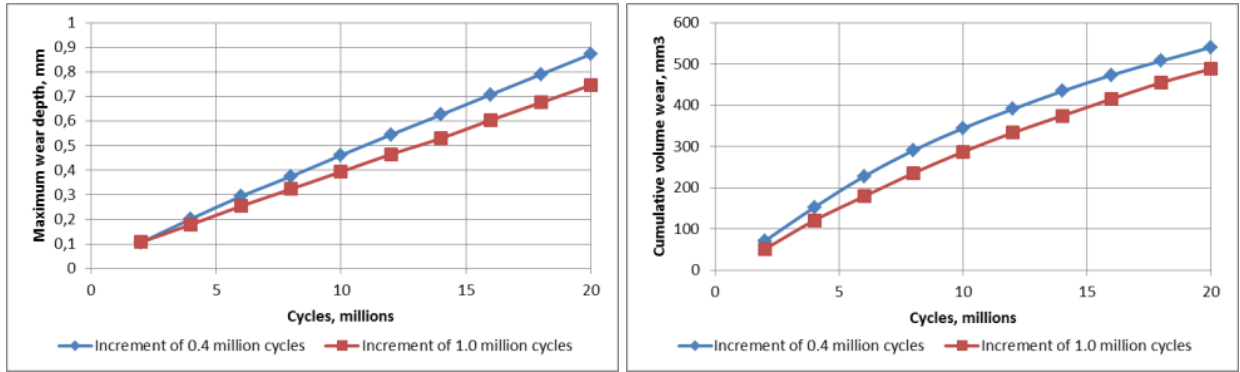

Fig. 10 Graphs on the impact of magnitude of increments of 0.4 and 1.0 million cycles on precision of calculations with a head diameter of $32 \mathrm{~mm}$ and according to ISO 14242-1 demands at variable wear factor by (5) in terms of the cumulative linear and volumetric wear 
The results are obtained in terms of the cumulative linear and volumetric wear of the cup surface at the parameters according to ISO 14242-1 test standard and during walking gait. Also, it is conducted for the head diameters of 32 and $28 \mathrm{~mm}$ at a constant wear factor of $1.066 \cdot 10^{-6}$ $\mathrm{mm}^{3} /(\mathrm{Nm})$ and of that variable by Eq. (5) up to 20 million cycles (see Figs. 11, 12, 13 and 14). In the studies at a variable wear factor, relationship (5) is adopted since initial dependence (3) corresponds to the material of the cup taken in this simulation.

\section{DISCUSSION}

Analysis of the obtained results which are illustrated in Figs. 11, 12, 13 and 14 can be expressed as follows. With the head diameter decreasing from $32 \mathrm{~mm}$ to $28 \mathrm{~mm}$, the volumetric wear is reduced at a constant and variable wear factor, according to ISO 14242-1 demands and during walking gait. Moreover, for smaller head diameter of 28 $\mathrm{mm}$ at a constant wear factor during walking gait, the linear wear increases by about $36 \%$ compared with the diameter of $32 \mathrm{~mm}$, which likely indicates a deeper penetration of the head into the cup, which can lead to more rapid disturbance of the joint biomechanics.

On the other hand, the linear wear according to ISO 14242-1 requirements for head diameters of $32 \mathrm{~mm}$ and $28 \mathrm{~mm}$ at a constant wear factor is almost the same $(1.78 \mathrm{~mm}$ and $1.85 \mathrm{~mm}$, respectively), and for diameter of $32 \mathrm{~mm}$, it practically coincides at the walking gait settings $(1.81 \mathrm{~mm})$. That is how it confirms the approximate equivalence of the walking gait conditions and of those according to ISO 14242-1 standard in this case.

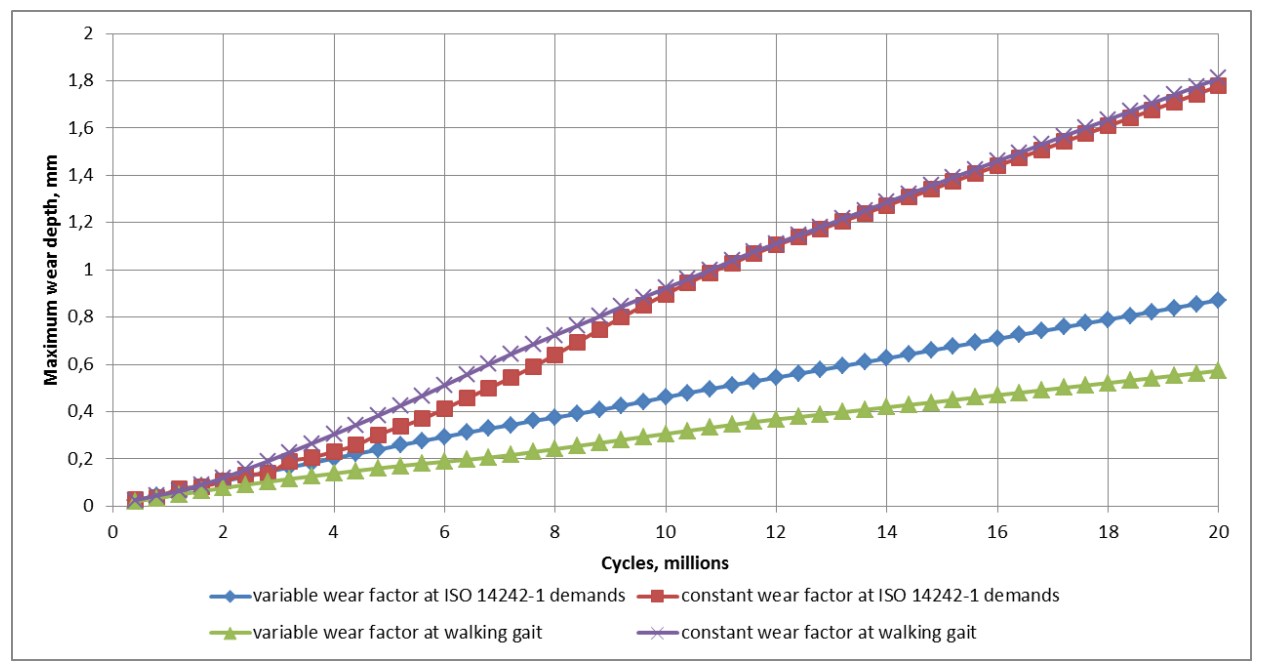

Fig. 11 The maximum cumulative linear wear (maximum wear depth) vs. time of the gait cycle according to ISO 14242-1 demands for a head diameter of $32 \mathrm{~mm}$ at a constant wear factor of $1.066 \cdot 10^{-6} \mathrm{~mm}^{3} /(\mathrm{Nm})$ and of that variable by (5) 


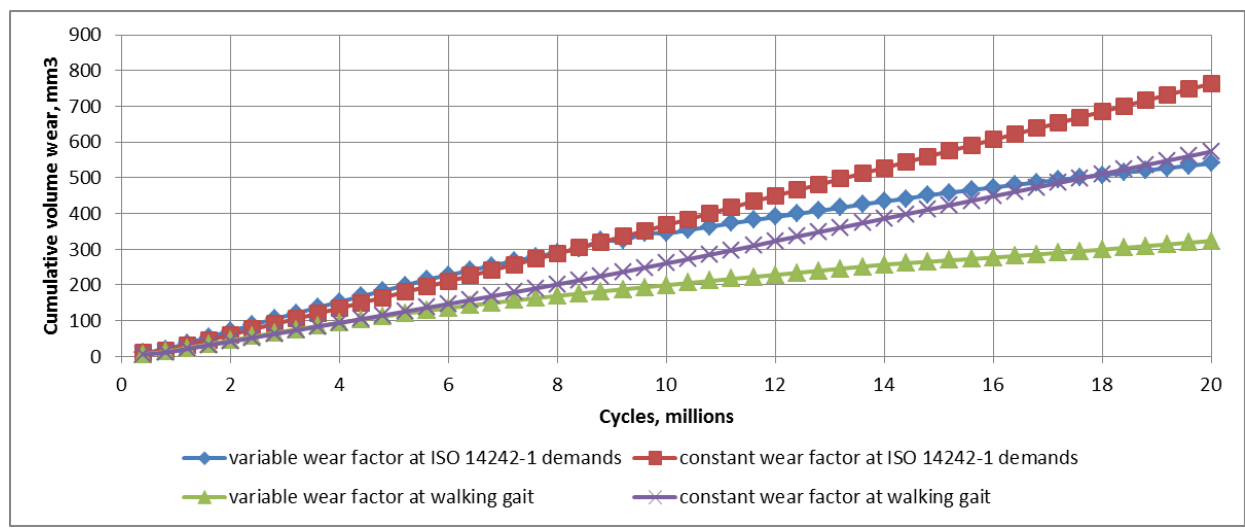

Fig. 12 The maximum cumulative volume wear vs. time of the gait cycle according to ISO 14242-1 demands for a head diameter of $32 \mathrm{~mm}$ at a constant wear factor of $1.066 \cdot 10^{-6} \mathrm{~mm}^{3} /(\mathrm{Nm})$ and of that variable by $(5)$

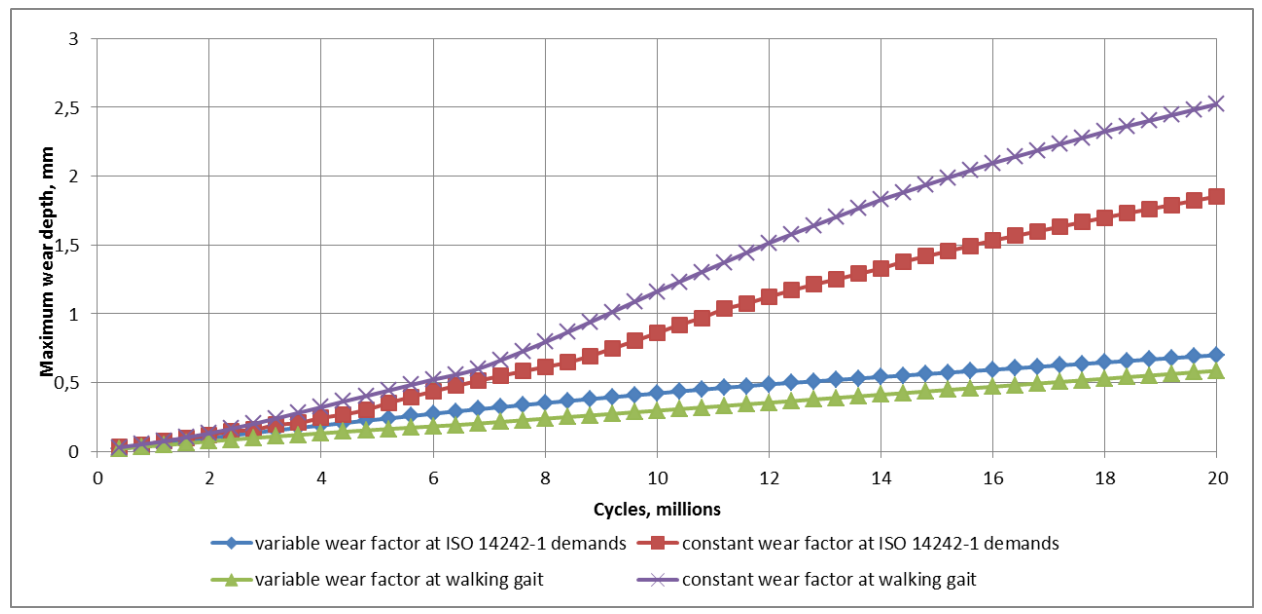

Fig. 13 The maximum cumulative linear wear (maximum wear depth) vs. time of the gait cycle according to ISO 14242-1 demands for a head diameter of $28 \mathrm{~mm}$ at a constant wear factor of $1.066 \cdot 10^{-6} \mathrm{~mm}^{3} /(\mathrm{Nm})$ and of that variable by (5)

The parameters of the ISO 14242-1 and walking gait have differences, mainly in the magnitude of the load (Fig. 4), where the maximum resultant load at ISO 14242-1 (3kN) is greater than for about $30 \%$ of the maximum load during walking gait $(2.23 \mathrm{kN})$ with approximately similar values of the angular parameters of displacement of the femoral head (Fig. 5,6). Thus, this fact is likely to impact the value of volumetric wear, which, for both head diameters indicated above at a constant and variable wear factor, is less at the walking gait conditions than at those of ISO 14242-1. 


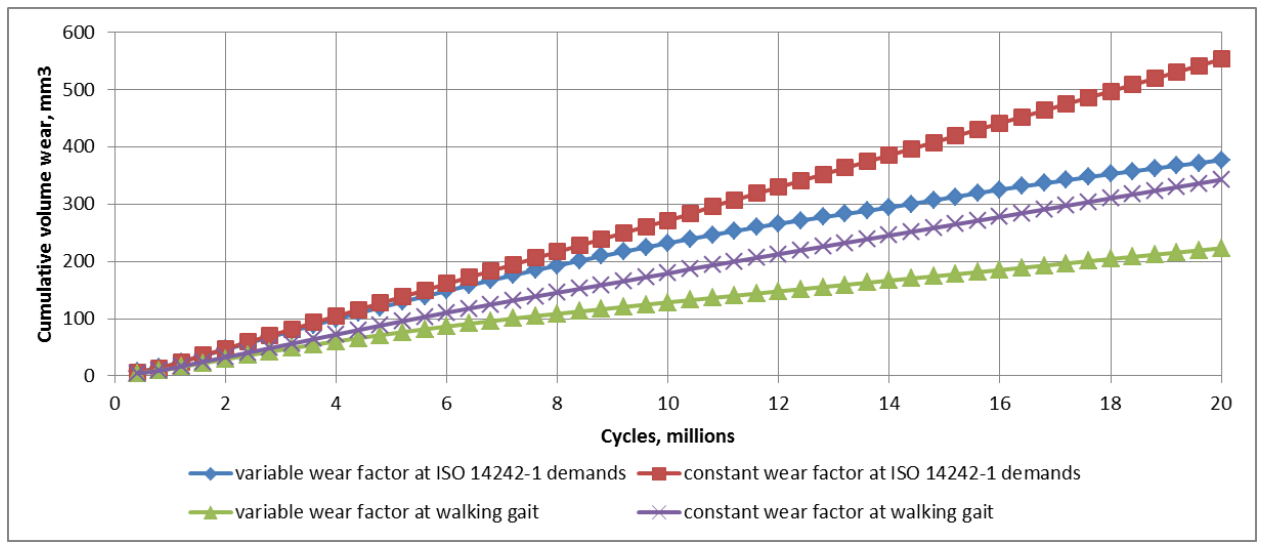

Fig. 14 The maximum cumulative volume wear vs. time of the gait cycle according to ISO 14242-1 demands for a head diameter of $28 \mathrm{~mm}$ at a constant wear factor of $1.066 \cdot 10^{-6} \mathrm{~mm}^{3} /(\mathrm{Nm})$ and of that variable by $(5)$

Simulation with a variable wear factor compared to that of the constant showed the following reduction of the amount of wear parameters at 20 million cycles for the head diameter of $32 \mathrm{~mm}$ : the linear wear at the ISO 14242-1 is approximately of 2 times, for the walking gait - in 3 times; the volumetric wear under the ISO 14242-1 is approximately of $40 \%$, for the walking gait - about of $77 \%$. The same stands for the head diameter of $28 \mathrm{~mm}$ : for the linear wear at the ISO 14242-1 approximately of 2.6 times, at the walking gait - about of 4.3 times; for the volumetric wear at the ISO 14242-1 approximately of $46 \%$, for the walking gait - about of 53\%. Such a large scale reduction in the linear wear determines not a very large decrease in the volumetric wear. This likely indicates that the wear surface in this case is redistributed not in depth but in breadth, which positively affects the lifespan of the implant, without greatly disturbing the joint biomechanics. Consequently, the simulation at a variable wear factor reflects a more real picture of the contact interaction in the implant couple, which is most clearly illustrated by the magnitude of the volumetric wear.

The nature of change of the cumulative linear wear in all the cases studied above is almost a linear relationship. But in the case of the head diameter of $28 \mathrm{~mm}$ at a constant wear factor for both ISO 14242-1 and for walking gait conditions, a small degree of nonlinearity is observed for the linear wear (Fig. 13). It also appears but more weakly at the head diameter of $32 \mathrm{~mm}$ in the interval up to 10 million cycles (Fig. 11). This could probably be due to the following factors. At the initial interval to 10 million cycles, there is an observed nonlinear increase in the depth of penetration of the head into the cup since the contact surface area has not increased enough to reduce the contact pressure efficiently. Moreover, the pressure reduction with the rise of cycle's number was noted and in [7]. With the deepening of the head, the surface contact area is increased; the contact pressure is reduced and slowed down and so is its penetration process. It has practically no impact on the almost linear nature of the change of volumetric wear since the profile of linear wear is concave-convex with respect to the straight line connecting the point of 0.4 and 20 million cycles, with a contraflexure in the middle of this range (10 
million). It can be assumed that this effect depends on the diameter head value and can be manifested more expressively when using its smaller standard diameter of $22 \mathrm{~mm}$.

The results of volumetric wear simulation of $573 \mathrm{~mm}^{3}$ at a constant wear factor for the walking gait conditions, in the case of the head diameter of $32 \mathrm{~mm}$, are in line up to $10 \%$ with the result $633 \mathrm{~mm}^{3}$ specified in [7] and up to $6 \%$ with a score of $541 \mathrm{~mm}^{3}$ in [6]. This, in turn, confirms the validity of this simulation.

\section{CONCLUSION}

The existing method of numerical wear simulation of the sliding couple in the spherical joint comprising a polymer element in terms of refining the calculation taking into account the parametric dependence of wear factor on the contact pressure according to ISO 14242-1 demands and of those during walking gait cycle, is improved. Also, studies are conducted at a constant wear factor in all indicated conditions and expanded to the implant head diameter of $28 \mathrm{~mm}$ in terms of the cumulative linear and volumetric wear. The use of the Archard equation in the form (5) makes it possible to clarify the simulation results only within a few percent, but it makes a more advanced calculation process at a variable wear factor. The developed method is a serious tool for the implementation of the more accurate initial qualifying analysis of design, materials and manufacturing process of the total hip replacement, and thus allows reducing the use of expensive experimental studies using simulators. Future studies can be focused on the accounting the daily activity of patients during wear simulation.

Acknowledgements: This work has been funded by the Ministry of Education and Science of the Russian Federation in the framework of the base part of state order in the field of scientific activity with the registration No.115041610028.

\section{REFERENCES}

1. Ingham, E., Fisher, J., 2000, Biological reactions to wear debris in total joint replacement, Proc Instn Mech Engrs, Part H: J Engineering in Medicine, 214(1), pp. 21-37.

2. Polyakov, A., Pakhaliuk, V., Kalinin, M., Kramar, V., Kolesova, M., Kovalenko, O., 2015, System analysis and synthesis of total hip joint endoprosthesis, Procedia Engineering, 100, pp. 530-538.

3. Poliakov, O.M., Pakhaliuk, V.I., Lazarev, V.B., Shtanko, P.K., Ivanov, Y.M., 2013, Stand and control system for wear testing of the spherical joints of vehicle suspension at complex loading conditions, IFAC Proceedings Volumes, 46(25), pp. 106-111.

4. Pakhaliuk, V., Poliakov, A., Kalinin, M., Bratan, S., 2016, Evaluating the impact and norming the parameters of partially regular texture on the surface of the articulating ball head in a total hip joint prosthesis, Tribology Online, 11(4), pp. 527-539.

5. Pakhaliuk, V.I., Polyakov, A.M., Kalinin, M.I., Kramar, V.A., 2015, Improving the finite element simulation of wear of total hip prosthesis' spherical joint with the polymeric component, Procedia Engineering, 100, pp. 539-548.

6. Maxian, T.A., Brown, T.D., Pedersen, D.R., Callaghan, J.J., 1996, A sliding-distance-coupled finite element formulation for polyethylene wear in total hip arthroplasty, J Biomechanics, 27, pp. 687-692.

7. Kang, L., Galvin, A.I., Jin, Z.M., Fisher, J., 2006, A simple fully integrated contact-coupled wear prediction for ultra-high weight polyethylene hip implants, Proc Instn Mech Engrs, Part H: J Engineering in Medicine, 220(1), pp. 35-46. 
8. Wu, J.S.S., Hung, J.P., Shu, C.S., Chen, J.H., 2003, The computer simulation of wear behavior appearing in total hip prosthesis, Computer Meth and Programs in Biomedicine, 70(1), pp. 81-91.

9. Wang, A., Essner, A., Klein, R., 2001, Effect of contact stress on friction and wear of ultra-high molecular weight polyethylene in total hip replacement, Proc Instn Mech Engrs, Part H: J Engineering in Medicine, 215(2), pp. 133-139.

10. Vassiliou, K., Unsworth, A., 2001, Is the wear factor in total joint replacements dependent on the nominal contact stress in ultra-high molecular weight polyethylene contacts? Proc Instn Mech Engrs, Part H: J Engineering in Medicine, 218(2), pp. 101-107.

11. Saikko, V., 2006, Effect of contact pressure on wear and friction of ultra-high molecular weight polyethylene in multidirectional sliding, Proc Instn Mech Engrs, Part H: J Engineering in Medicine, 220(7), pp. 723-731. 